\title{
On the Performance of Full-Duplex Multi-Relay Channels with DF Relays
}

\author{
Qiang Li, Member, IEEE, Shangjie Feng, Xiaohu Ge, Senior Member, IEEE, \\ Guoqiang Mao, Senior Member, IEEE, and Lajos Hanzo, Fellow, IEEE
}

\begin{abstract}
In this paper, we consider a two-hop full-duplex multi-relay channel (FD-MRC) for exploiting the high spectral efficiency of FD systems and the diversity gains of multirelay systems. Employing a decode-and-forward (DF) relaying protocol, the single best relay is selected from those relays that have successfully decoded the source message, which then helps forward the source message to the destination. Meanwhile, a new message is broadcasted by the source and all the $N$ relays, including the selected best one, attempt to decode this new message. In view of the effect of both inter-relay interference and self-interference (SI), a one-step correlation exists between the relay decoding results in successive time slots. Then a Markov chain-based analytical model is utilized for analyzing the exact outage probability of FD-MRC. Furthermore, a closeto-full diversity order of $(N-1+\mu)$ is analytically proved to be attainable for FD-MRC, where $\mu$ is a factor characterizing the efficiency of SI mitigation.
\end{abstract}

Index Terms-Full-duplex relays, relay selection, interference, outage probability, diversity.

\section{INTRODUCTION}

Full-duplex (FD) techniques have been introduced in recent $5 \mathrm{G}$ standard proposals as an appealing technique of significantly enhancing the attainable spectral efficiency (SE) of communication systems [1]-[3]. Although theoretically a nearly two-fold SE improvement can be accomplished by concurrent transmission and reception, the performance of FD systems could be severely compromised by the selfinterference (SI) that leaks from the transmit antenna of the FD device to its own receive antenna [4]. In the face of excessive SI, the FD system may even perform worse than its half-duplex (HD) counterpart. Thus in order to unleash the potential of FD

Copyright (c) 2015 IEEE. Personal use of this material is permitted. However, permission to use this material for any other purposes must be obtained from the IEEE by sending a request to pubs-permissions@ieee.org.

The authors would like to acknowledge the support from the National Natural Science Foundation of China (NSFC) under grants 61461136004, NFSC Major International Joint Research Project under grant 61210002, the Hubei Provincial Science and Technology Department under Grant 2016AHB006 and the Fundamental Research Funds for the Central Universities under grant 2015XJGH011. This research is partially supported by EU FP7-PEOPLEIRSES 610524, China International Joint Research Center of Green Communications and Networking (No. 2015B01008).

Qiang Li, Shangjie Feng, and Xiaohu Ge (corresponding author) are with the School of Electronic Information and Communications, Huazhong University of Science and Technology, Wuhan 430074, China. Emails: \{qli_patrick, sjfeng,xhge\}@hust.edu.cn.

Guoqiang Mao is with University of Technology Sydney and National ICT Australia, Sydney, NSW 1466, Australia. Email: guoqiang.mao@uts.edu.au.

Lajos Hanzo (corresponding author) is with the School of Electronics and Computer Science, University of Southampton, Southampton SO17 1BJ, U.K. Email: 1h@ecs.soton.ac.uk. techniques, it is a widely maintained consensus that the SI has to be suppressed [5], [6].

To avoid severe SI, a sophisticated hybrid FD/HD relaying scheme was proposed in [7] that adaptively switches between the FD and HD modes based on the instantaneous SI. An optimal power control scheme was proposed in [8] for the FD relay to mitigate its SI, which achieves a higher diversity order than full-power transmissions. Various FD relaying schemes were investigated in [9], where a codeword spans over multiple independent channel realizations. This codeword expansion technique benefits from time diversity that is shown to be dependent on the efficiency of the SI cancelation. Additionally, the SI can also be mitigated by employing multiple antennas, see e.g., [10], [11] and the references therein.

In order to exploit the extra diversity gains facilitated by multiple relays [12]-[17], multi-FD-relay aided systems have been studied [18], [19]. An optimal relay selection scheme that requires global channel state information (CSI) was proposed in [18] for amplify-and-forward (AF) FD relay channels. Approximated outage probabilities were derived in the high signal-to-noise ratio (SNR) regime, where no diversity gains can be achieved without an effective interference canceller. The hybrid FD/HD scheme of [7] achieved diversity gains as demonstrated in [18], but at the expense of some spectral efficiency loss compared to ideal FD relaying. In [19], a bufferaided FD relaying scheme was proposed for AF relays with iterative successive interference cancelation performed at the destination. A full diversity order was achieved while at the cost of increased delays.

Motivated by the aforementioned studies, in this paper we consider a FD multi-relay channel (FD-MRC) with decodeand-forward (DF) relays for exploiting the high spectral efficiency of FD systems while reducing their vulnerability to SI. Each time the source finishes transmitting a new message, a candidate set is formed by those relays that have successfully decoded this message. Then, from this set the single best relay having the highest relay-destination channel quality is selected [12], [13]. Then at the instant of the relay's transmission, a new message is transmitted from the source and all the $N$ FD relays attempt to decode it. Due to the simultaneous transmissions from the selected relay and the source, there exists an interrelay interference (IRI) in addition to the SI of the selected FD relay. The main contributions of this treatise are summarized as follows.

- Accounting for the effects of both IRI as well as SI, and for the correlation of the relay decoding results in succes- 


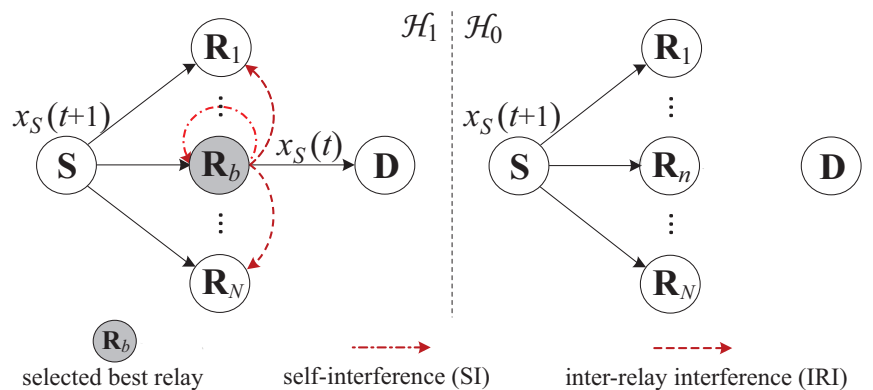

Fig. 1. A full-duplex multi-relay channel (FD-MRC) with DF relays.

sive time lots, the exact outage probability of FD-MRC is obtained by using a Markov chain-based analytical model [20], [21], which points beyond the state-of-theart where the attainable diversity order is characterized without explicitly analyzing the outage probability [14].

- Upon relying on a factor $0 \leq \mu \leq 1$ indicating the efficiency of SI mitigation, a close-to-full diversity order of $(N-1+\mu)$ is shown to be attained for FD-MRC, which is higher than that of the existing spectrallyefficient HD relaying schemes [14], [17] and that of the FD relaying schemes associated with AF relays [18].

- Comparisons with existing HD/FD schemes are conducted under various conditions and system parameters. The performance gains achieved by the considered FDMRC with DF relays are demonstrated.

\section{SYSTEM MODEL}

As shown in Fig. 1, we consider a two-hop FD-MRC with $N$ FD DF relays $\mathbf{R}_{i}, i \in \mathcal{N}=\{1,2, \cdots, N\}$. Due to a large distance between the source $\mathbf{S}$ and destination $\mathbf{D}$, the direct $\mathbf{S} \rightarrow \mathbf{D}$ channel is sufficiently weak that can be ignored [9]. To focus our attention on the performance analysis of FD-MRC, the symmetric network setup of [12]-[17] is assumed, where the relay channels $\mathbf{S} \rightarrow \mathbf{R}_{i}$ and $\mathbf{R}_{i} \rightarrow \mathbf{D}$, for $i \in \mathcal{N}$, experience independent and identical Rayleigh fading, respectively. Then we have the channel coefficients $h_{s, i} \sim \mathcal{C N}\left(0, \delta_{s, r}^{-1}\right)$ and $h_{i, d} \sim \mathcal{C N}\left(0, \delta_{r, d}^{-1}\right)$, with the corresponding channel power gains given by $\gamma_{s, i}=\left|h_{s, i}\right|^{2}$ and $\gamma_{i, d}=\left|h_{i, d}\right|^{2}$, which follow exponential distributions $\gamma_{s, i} \sim \exp \left(\delta_{s, r}\right)$ and $\gamma_{i, d} \sim$ $\exp \left(\delta_{r, d}\right)$, respectively.

In the FD-MRC considered, a new source symbol $x_{S}(t)$ is originated from $\mathbf{S}$ in each time slot $t$ and all $N$ FD relays attempt to decode it. For simplicity, the transmit powers at $\mathbf{S}$ and the relays are assumed to be equal and are denoted as $\rho$ [9]. The additive white Gaussian noise is denoted as $n_{r}$, where $r \in\{\mathcal{N}, d\}$, and is assumed to be of zero mean and of unit variance. Depending on the decoding status of the relays, a successful decoding set $\mathcal{D}$ is defined that contains all relays that have successfully decoded $x_{S}(t)$, where $\mathcal{D} \subseteq \mathcal{N}$.

If $\mathcal{D} \neq \emptyset$, which is defined as state $\mathcal{H}_{1}$, then the single best relay $\mathbf{R}_{b}$ having the highest instantaneous relay-destination channel gains is selected. Specifically, channel-reciprocity is assumed [12]-[17], where the destination $\mathbf{D}$ first broadcasts a clear-to-send (CTS) control signal at the end of each time slot. Upon receiving CTS, each relay $\mathbf{R}_{i}, i \in \mathcal{D}$ triggers a countdown timer with an initial value that is inversely proportional to the estimated channel quality from $\mathbf{D}$. Then the countdown timer at $\mathbf{R}_{b}$, where $b=\arg \max _{i \in \mathcal{D}}\left\{\gamma_{i, d}\right\}$, will expire first. $\mathbf{R}_{b}$ is then triggered to broadcast a readyto-send (RTS) signal to notify the rest of the relays as well as the destination. It is assumed that these control signaling exchanges involved in relay selection are completed instantly such that a single best relay can always be selected without collision [22]. Then in the subsequent time slot $(t+1), \mathbf{R}_{b}$ forwards the decoded symbol $x_{S}(t)$ concurrently with $\mathbf{S}$ 's transmission of a new symbol $x_{S}(t+1)$, as illustrated in Fig. 1. Thus the signals received at $\mathbf{R}_{i}$ and $\mathbf{D}$ are given by

$$
\begin{aligned}
y_{i}(t+1) & =h_{s, i} \sqrt{\rho} x_{S}(t+1) \\
& +h_{b, i} \sqrt{\rho} x_{S}(t)+n_{i}(t+1), \quad i \in \mathcal{N} \backslash b,(1) \\
y_{d}(t+1) & =h_{b, d} \sqrt{\rho} x_{S}(t)+n_{d}(t+1)
\end{aligned}
$$

respectively, where $h_{b, i} \sim \mathcal{C N}\left(0, \delta_{\text {IRI }}^{-1}\right), \forall b, i \in \mathcal{N}$ and $b \neq$ $i$, denotes the IRI channel coefficient. Provided that the SI cannot be fully eliminated due to practical imperfections [1][6], the transmitted SI power is assumed to scale with the relay transmit power by obeying an exponent $\mu$, i.e., we have $\rho_{\mathrm{SI}}=\rho^{1-\mu}$, where $0 \leq \mu \leq 1$ characterizes the efficiency of SI cancellation [9]. Then the received signal at $\mathbf{R}_{b}$ subjected to SI is given by

$$
\begin{aligned}
y_{b}(t+1) & =h_{s, b} \sqrt{\rho} x_{S}(t+1) \\
& +h_{b, b} \sqrt{\rho_{\mathrm{SI}}} x_{S}(t)+n_{b}(t+1),
\end{aligned}
$$

where $h_{b, b} \sim \mathcal{C N}\left(0, \delta_{\mathrm{SI}}^{-1}\right)$ denotes the SI channel coefficient. It is worth noting that there exists some residual SI even when $\mu=1$. This represents that even if the SI cannot be perfectly removed, the residual SI after its mitigation has a negligible effect as the transmit power increases. Since we focus out attention on the performance analysis under a given residual SI strength, no specific SI mitigation schemes are considered.

If however $\mathcal{D}=\emptyset$, which is defined as state $\mathcal{H}_{0}$, then no relay is selected and all the $N$ relays listen to the new source symbol transmitted in slot $(t+1)$, as illustrated in Fig. 1. Then an outage is declared for $x_{S}(t)$ since no signal will be received by $\mathbf{D}$. The corresponding signal received at $\mathbf{R}_{i}$ is given by

$$
y_{i}(t+1)=h_{s, i} \sqrt{\rho} x_{S}(t+1)+n_{i}(t+1), \quad i \in \mathcal{N} .
$$

\section{Outage Performance Analysis}

\section{A. A Markov Chain-Based Analytical Model}

Based on the system model described in Section II, there exists a correlation between the relay success/failure status in successive time slots. For instance, conditioned on state $\mathcal{H}_{1}$ in time slot $t, \mathbf{R}_{i}$ attempts to decode $x_{S}(t+1)$ subject to the IRI arriving from $\mathbf{R}_{b}$, while $\mathbf{R}_{b}$ attempts to decode $x_{S}(t+1)$ that suffers from SI, as formulated in (1) and (3), respectively. On the other hand, conditioned on state $\mathcal{H}_{0}$ in time slot $t$, no relay is selected and all the $N$ relays attempt to decode the new 
source symbol $x_{S}(t+1)$ free of interference, as given in (4). This means that depending on whether state $\mathcal{H}_{1}$ or $\mathcal{H}_{0}$ occurs in slot $t$, the relays may or may not suffer from interference, thus leading to different decoding results for $x_{S}(t+1)$ in time slot $(t+1)$. In other words, this imposes a dependency of the decoding status of $x_{S}(t+1)$ on that of $x_{S}(t)$ [20], [21].

To model this one-step correlation, we propose using a two-state Markov chain for characterizing the state transitions between $\mathcal{H}_{0}$ and $\mathcal{H}_{1}$ in successive time slots. For ease of exposition, we define $\pi_{0}$ and $\pi_{1}$ as the long-term steady-state probabilities of $\mathcal{H}_{0}$ and $\mathcal{H}_{1}$ respectively, and let $P_{00}, P_{01}$, $P_{10}$, and $P_{11}$ denote the corresponding transition probabilities. Then, the stationary probabilities of $\mathcal{H}_{1}$ and $\mathcal{H}_{0}$ satisfy the following relationship

$$
\pi_{0} P_{00}+\pi_{1} P_{10}=\pi_{0}, \pi_{0} P_{01}+\pi_{1} P_{11}=\pi_{1}, \pi_{0}+\pi_{1}=1,
$$

from which $\pi_{0}$ and $\pi_{1}$ can be respectively obtained as

$$
\pi_{0}=\frac{P_{10}}{P_{10}+P_{01}}, \quad \pi_{1}=\frac{P_{01}}{P_{10}+P_{01}} .
$$

It is worth noting at this stage that with probability $\pi_{0}$, an outage occurs because no signal will be forwarded to $\mathbf{D}$. On the other hand, with probability $\pi_{1}$ that there is at least one relay successfully decodes the source message, there also exist a non-zero outage probability during the $\mathbf{R}_{b} \rightarrow \mathbf{D}$ transmission.

\section{B. Decoding Status of the Relays and Transition Probabilities}

In order to obtain $\pi_{0}$, next we analyze the transition probabilities $P_{00}, P_{01}, P_{10}, P_{11}$ from slot $t$ to slot $(t+1)$.

1) Conditioned on State $\mathcal{H}_{0}$ in Slot $t$ : From $(4), x_{S}(t)$ is discarded and all relays attempt to decode $x_{S}(t+1)$ in slot $(t+$ 1). Thus with a target rate of $R_{0}$ bits/slot/Hz, the probability that $\mathbf{R}_{i}$ successfully decodes $x_{S}(t+1)$ is given by

$$
P_{01}^{(i)}=\operatorname{Pr}\left\{\log _{2}\left(1+\gamma_{s, i} \rho\right) \geq R_{0}\right\}=e^{-\frac{\delta_{s, r} \gamma_{0}}{\rho}},
$$

where $\gamma_{0}=2^{R_{0}}-1$. Thus we have $P_{01}=1-P_{00}$, where

$$
P_{00}=\prod_{i \in \mathcal{N}}\left(1-P_{01}^{(i)}\right)=\left(1-e^{-\frac{\delta_{s, r} \gamma_{0}}{\rho}}\right)^{N} .
$$

2) Conditioned on State $\mathcal{H}_{1}$ in Slot t: From (1) and (3), a single best relay $R_{b}$ is selected that imposes IRI on the remaining $(N-1)$ relays while causing SI to itself. Next we analyze the decoding performance of these relays, respectively.

Lemma 1: From (1), due to the simultaneous transmissions from $\mathbf{S}$ and $\mathbf{R}_{b}$, a multiple-access channel (MAC) is seen at $\mathbf{R}_{i}$, where $i \in \mathcal{N} \backslash b$. Then the probability $P_{11}^{(i)}$ that $\mathbf{R}_{i}$ successfully decodes $x_{S}(t+1)$ is analytically formulated in (9).

(9) can be obtained by exploiting the capacity region of the conventional MAC, where both symbols $x_{S}(t+1)$ and $x_{S}(t)$ are decoded by $\mathbf{R}_{i}$, together with the capacity region, where only the desired symbol $x_{S}(t+1)$ is decoded [23]. The details are omitted here due to space limitation.
On the other hand, as seen in (3), the probability that $\mathbf{R}_{b}$ successfully decodes $x_{S}(t+1)$ is given by

$$
\begin{aligned}
P_{11}^{(b)} & =\operatorname{Pr}\left\{\log _{2}\left(1+\frac{\gamma_{s, b} \rho}{\gamma_{b, b} \rho^{1-\mu}+1}\right) \geq R_{0}\right\} \\
& =\operatorname{Pr}\left\{\gamma_{s, b} \geq \gamma_{b, b} \gamma_{0} \rho^{-\mu}+\gamma_{0} \rho^{-1}\right\} .
\end{aligned}
$$

For this inequality involving random variables $\gamma_{s, b}$ and $\gamma_{b, b}$, the probability can be derived using the following integration

$$
\begin{aligned}
& \int_{0}^{\infty} f\left(\gamma_{b, b}\right)\left[\int_{\gamma_{b, b} \gamma_{0} \rho^{-\mu}+\gamma_{0} \rho^{-1}}^{\infty} f\left(\gamma_{s, b}\right) d \gamma_{s, b}\right] d \gamma_{b, b} \\
= & \frac{\delta_{\mathrm{SI}} e^{-\frac{\delta_{s, r} \gamma_{0}}{\rho}}}{\delta_{s, r} \rho^{-\mu} \gamma_{0}+\delta_{\mathrm{SI}}}
\end{aligned}
$$

where $f\left(\gamma_{s, b}\right)=\delta_{s, r} e^{-\delta_{s, r} \gamma_{s, b}}$ and $f\left(\gamma_{b, b}\right)=\delta_{\mathrm{SI}} e^{-\delta_{\mathrm{SI}} \gamma_{b, b}}$ denote their respective probability density functions.

From (9)-(11), we have $P_{11}=1-P_{10}$, where

$$
P_{10}=\left(1-P_{11}^{(b)}\right) \prod_{i \in \mathcal{N} \backslash b}\left(1-P_{11}^{(i)}\right) .
$$

From the above analysis, all major performance-impacting factors of whether a relay is suffering from IRI, or SI, or free of interference are considered for forming a candidate relay set $\mathcal{D}$. Furthermore, it is worth noting that compared to equal transmission power at the source and relays, performance gains can be expected with an adaptive power control at the selected relay or a power allocation between the source and relay, which is delegated to our future work due to space limitation.

\section{End-to-End Outage Probability}

1) Conditioned on State $\mathcal{H}_{0}$ in Slot $t$ : Since no signal will be forwarded to $\mathbf{D}$, an outage is declared for $x_{S}(t)$.

2) Conditioned on State $\mathcal{H}_{1}$ in Slot $t$ : The single best relay $\mathbf{R}_{b}$ is selected from $\mathcal{D}$ for forwarding $x_{S}(t)$ in slot $(t+1)$, as given in (2). Then, taking into account the cardinality of the successful decoding set $|\mathcal{D}|$, the achievable rate of decoding $x_{S}(t)$ at $\mathbf{D}$ is given by $R_{d}=\log _{2}\left(1+\max _{i \in \mathcal{D}}\left\{\gamma_{i, d}\right\} \rho\right)$, whose cumulative distribution function can be expressed as

$$
F_{R_{d}}\left(R_{0}\right)=\sum_{l=1}^{N} \operatorname{Pr}\{|\mathcal{D}|=l\} F_{R_{d}}\left(R_{0}|| \mathcal{D} \mid=l\right),
$$

where

$$
F_{R_{d}}\left(R_{0}|| \mathcal{D} \mid=l\right)=\left(1-e^{-\frac{\delta_{r, d} \gamma_{0}}{\rho}}\right)^{l}
$$

denotes the probability that none of the $|\mathcal{D}|=l$ relays can successfully deliver $x_{S}(t)$ to $\mathbf{D}$.

Depending on whether the state $\mathcal{H}_{1}$ in slot $t$ was reached from the previous state $\mathcal{H}_{0}$ or $\mathcal{H}_{1}$ in slot $t-1$, we have

$$
\operatorname{Pr}\{|\mathcal{D}|=l\}=\overbrace{\pi_{0} \operatorname{Pr}\left\{|\mathcal{D}|=l \mid \mathcal{H}_{0}\right\}}^{\mathcal{H}_{0} \text { in slot } t-1}+\overbrace{\pi_{1} \operatorname{Pr}\left\{|\mathcal{D}|=l \mid \mathcal{H}_{1}\right\}}^{\mathcal{H}_{1} \text { in slot } t-1},
$$

where $\operatorname{Pr}\left\{|\mathcal{D}|=l \mid \mathcal{H}_{0}\right\}=\left(\begin{array}{c}N \\ l\end{array}\right)\left(P_{01}^{(i)}\right)^{l}\left(1-P_{01}^{(i)}\right)^{N-l}$ and $\operatorname{Pr}\left\{|\mathcal{D}|=l \mid \mathcal{H}_{1}\right\}=\left(\begin{array}{c}N-1 \\ l\end{array}\right)\left(P_{11}^{(i)}\right)^{l}\left(1-P_{11}^{(i)}\right)^{N-1-l}(1-$ $\left.P_{11}^{(b)}\right)+\left(\begin{array}{c}N-1 \\ l-1\end{array}\right)\left(P_{11}^{(i)}\right)^{l-1}\left(1-P_{11}^{(i)}\right)^{N-l} P_{11}^{(b)}$, respectively. 


$$
P_{11}^{(i)}=1-\frac{\delta_{\mathrm{IRI}}\left[1-e^{-\frac{\left(\delta_{s, r}\left(\gamma_{0}+1\right)+\delta_{\mathrm{IRI}}\right) \gamma_{0}}{\rho}}\right]-\delta_{s, r}\left[1-e^{-\frac{\left(\delta_{\mathrm{IRI}}\left(\gamma_{0}+1\right)+\delta_{s, r}\right) \gamma_{0}}{\rho}}\right]}{\delta_{\mathrm{IRI}}-\delta_{s, r}}+\frac{\delta_{\mathrm{IRI}} e^{-\frac{\delta_{s, r} \gamma_{0}}{\rho}}\left[1-e^{-\frac{\left(\delta_{\mathrm{IRI}}+\delta_{s, r} \gamma_{0}\right) \gamma_{0}}{\rho}}\right]}{\delta_{\mathrm{IRI}}+\delta_{s, r} \gamma_{0}} .
$$

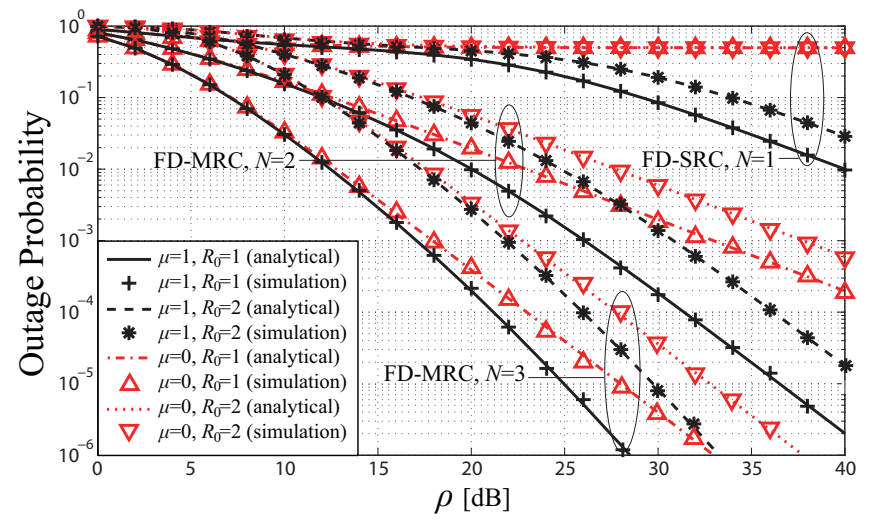

Fig. 2. Outage performance comparisons between FD-MRC and FD-SRC.

From the above analysis, taking into account the stochastic transitions between $\mathcal{H}_{0}$ and $\mathcal{H}_{1}$ in successive time slots, the overall average outage probability of FD-MRC is obtained as

$$
O_{p}=\pi_{0}+F_{R_{d}}\left(R_{0}\right) .
$$

\section{DiVERSiTy ANALYSis}

In this section, we investigate the diversity order of FDMRC, as formulated in Theorem 1.

Theorem 1: For the FD-MRC under consideration, where a single best relay is selected from $N$ FD relays to opportunistically forward the information originated from $\mathbf{S}$ to $\mathbf{D}$, a closeto-full diversity order of $(N-1+\mu)$ can be asymptotically achieved in the high-SNR regime, where $\mu$ is a factor that characterizes the quality of SI mitigation.

Please refer to Appendix A for the proof.

\section{Simulation Results}

In this section, simulation results are presented for characterizing the performance of the FD-MRC considered. For ease of illustration, we let $R_{0}=1 \mathrm{bit} / \mathrm{slot} / \mathrm{Hz}, \delta_{s, r}^{-1}=0 \mathrm{~dB}$, $\delta_{r, d}^{-1}=0 \mathrm{~dB}, \delta_{\mathrm{IRI}}^{-1}=10 \mathrm{~dB}$, and $\delta_{\mathrm{SI}}^{-1}=20 \mathrm{~dB}$ respectively, unless otherwise specified. For comparisons, the performance of the following two benchmark cases are also presented.

1) FD single-relay channel (FD-SRC) with DF relaying.

2) HD multi-relay channel (HD-MRC) with relay selection.

A performance comparison between FD-MRC and FD-SRC is shown in Fig. 2. With more relays available, since the source symbol can be delivered to the destination through more paths potentially, a higher diversity order of $(N-1+\mu)$ can be achieved by FD-MRC asymptotically, which results in an obvious performance gain over FD-SRC. For an extreme scenario where there is no SI mitigation, i.e., $\mu=0$, it is observed that an error floor is experienced by FD-SRC. Whereas reasonably good performances can still be achieved

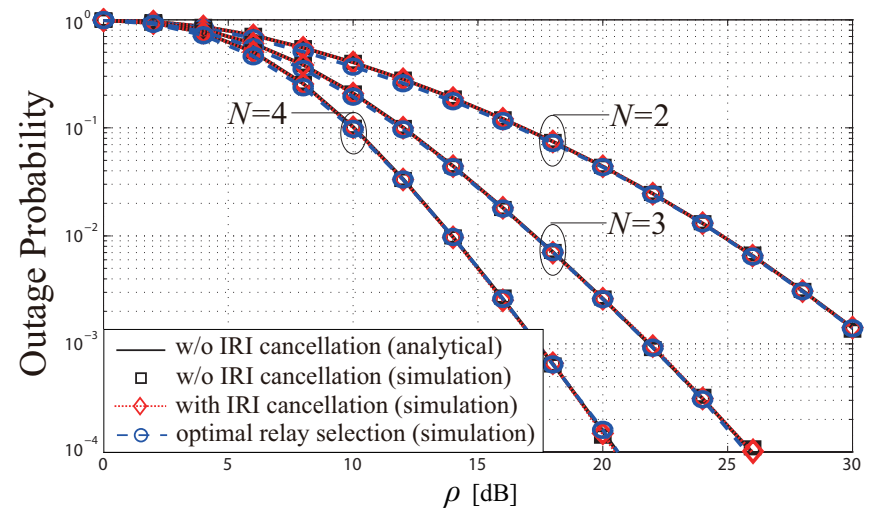

Fig. 3. Outage probability of FD-MRC when $\mu=1$ and $R_{0}=2$ bits/slot/Hz.

for FD-MRC with a diversity order of $(N-1)$. On the contrary, with perfect SI mitigation where $\mu=1$, it is observed that a full diversity order of $N$ is achieved for FD-MRC asymptotically. Furthermore, with an increase in $R_{0}$, it is observed that the overall outage performance is degraded, while attaining the same diversity order asymptotically.

As illustrated in Fig. 3, we also present the performance of FD-MRC in conjunction with IRI cancellation, where the IRI imposed by $\mathbf{R}_{b}$ can be perfectly removed from relay $\mathbf{R}_{i}$, for $i \in\{\mathcal{D} \backslash b\}$, by using the decoded source message as side information. It is observed that the corresponding system outage probability provides a tight performance upper bound of the case without IRI cancellation. Furthermore, an optimal relay selection scheme is simulated by striking a balance between the source-relay and relay-destination transmissions that are coupled by the SI and IRI. By selecting the specific relay that maximizes the cardinality of the candidate relay set $|\mathcal{D}|$ in slot $(t+1)$ at the prerequisite of successfully delivering the source message $x_{S}(t)$ to the destination, it is observed that a slightly better outage performance is achieved by the optimal relay selection scheme than by the benchmark relay selection scheme, when both have the same diversity orders.

A performance comparison between FD-MRC and HDMRC is shown in Fig. 4. It is observed a higher diversity order can be achieved for FD-MRC by deploying more relays or by implementing a more efficient SI mitigation, which results in an obvious performance improvement. On the other hand, although HD-MRC achieves a full diversity order of $N$, this higher diversity order of HD-MRC may not translate into high performance gains until the SNR becomes sufficiently high. For instance, HD-MRC outperforms FD-MRC at around $\rho=14,20,26 \mathrm{~dB}$ for $N=2,3,4$, respectively.

We infer from the above observations that FD-MRC is more robust than FD-SRC in that even when suffering from severe 


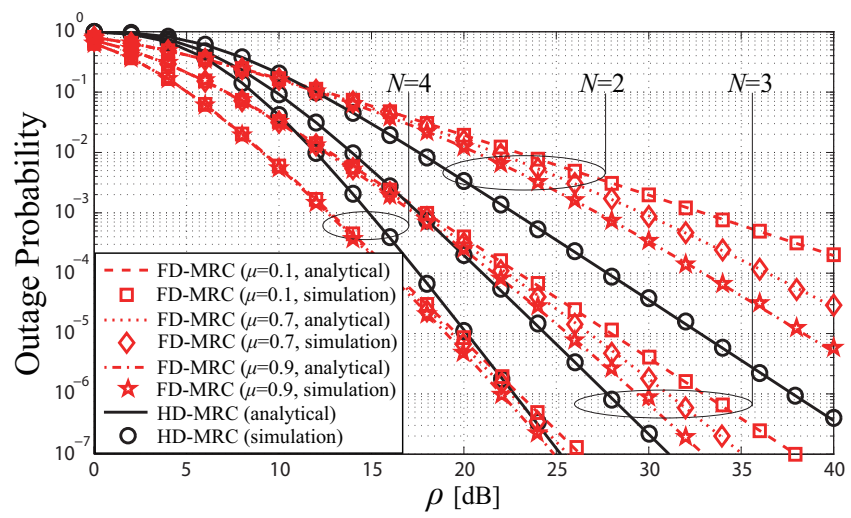

Fig. 4. Outage performance comparisons between FD-MRC and HD-MRC.

residual SI, a good performance can be achieved for FD-MRC by deploying multiple relays. Furthermore, under practical situations of low to modest SNR values, a better outage performance can be achieved by FD-MRC than HD-MRC, albeit at a higher complexity due to interference mitigation.

\section{CONClusions}

In this paper, a FD-MRC was investigated with the objective of achieving the high spectral efficiency of FD systems and the diversity gains of multi-relay systems. By using a Markov chain-based analytical technique for modeling the dependency between the relays' decoding status in successive time slots, both the end-to-end outage probability and the diversity order of FD-MRC were analyzed and derived in closed forms. Our results demonstrate the performance gains of FD-MRC over existing HD/FD schemes, where a high spectral efficiency is achieved by FD-MRC that is robust to the residual SI.

\section{APPENDIX A \\ Proof OF THEOREM 1}

For ease of analysis, we define an operator $\doteq$ such that for a probability $P(\rho)$, if $-\lim _{\rho \rightarrow \infty} \frac{\log P(\rho)}{\log \rho}=\alpha$, then $P(\rho) \doteq \rho^{-\alpha}$ [9]. This means that $P(\rho)$ decreases at a rate of $\rho^{-\alpha}$ when $\rho \rightarrow \infty$, thus achieving a diversity order of $\alpha$. Similarly, for a positive value $\beta$, since $-\lim _{\frac{1}{\beta} \rightarrow \infty} \frac{\log \left(1-e^{-\beta}\right)}{\log \left(\frac{1}{\beta}\right)}=1$ based on L'Hospital's Rule, we have $1-e^{-\beta} \stackrel{\doteq}{=} \beta$. Using this transformation, we have from (11)

$$
\begin{aligned}
1-P_{11}^{(b)} & =\frac{\delta_{s, r} \rho^{-\mu} \gamma_{0}+\delta_{\mathrm{SI}}\left(1-e^{-\frac{\delta_{s, r} \gamma_{0}}{\rho}}\right)}{\delta_{s, r} \rho^{-\mu} \gamma_{0}+\delta_{\mathrm{SI}}} \\
& \doteq \frac{\delta_{s, r} \rho^{-\mu} \gamma_{0}+\delta_{\mathrm{SI}} \delta_{s, r} \gamma_{0} \rho^{-1}}{\delta_{s, r} \rho^{-\mu} \gamma_{0}+\delta_{\mathrm{SI}}} \doteq \rho^{-\mu}
\end{aligned}
$$

From (9), similarly we have $1-e^{-\frac{\left(\delta_{s, r}\left(\gamma_{0}+1\right)+\delta_{\text {IRI }}\right) \gamma_{0}}{\rho}} \doteq \rho^{-1}$, $1-e^{-\frac{\left(\delta_{\mathrm{IRI}}\left(\gamma_{0}+1\right)+\delta_{s, r}\right) \gamma_{0}}{\rho}} \doteq \rho^{-1}$, and $1-e^{-\frac{\left(\delta_{\mathrm{IRI}}+\delta_{s, r} \gamma_{0}\right) \gamma_{0}}{\rho}} \doteq \rho^{-1}$, thus obtaining

$$
1-P_{11}^{(i)} \doteq \rho^{-1}
$$

Substituting (17), (18) into (12), we have

$$
P_{10} \doteq \rho^{-\mu} \cdot\left(\rho^{-1}\right)^{N-1} \doteq \rho^{-(N-1+\mu)} .
$$

Similarly, since $1-P_{01}^{(i)} \doteq \rho^{-1}$ from (7), we have from (8)

$$
P_{00} \doteq\left(\frac{\delta_{s, r} \gamma_{0}}{\rho}\right)^{N} \doteq \rho^{-N} \text {. }
$$

Since $\pi_{0}=\frac{P_{10}}{P_{10}+P_{01}}=\frac{P_{10}}{1+\left(P_{10}-P_{00}\right)}$ as given in (6), we have

$$
\pi_{0} \doteq P_{10} \doteq \rho^{-(N-1+\mu)} .
$$

Furthermore, we have from (14) and (15)

$$
\begin{aligned}
F_{R_{d}}\left(R_{0}|| \mathcal{D} \mid=l\right) & \doteq \rho^{-l}, \\
\operatorname{Pr}\{|\mathcal{D}|=l\} & \doteq \rho^{-(N-1-l+\mu)},
\end{aligned}
$$

respectively. Then substituting (22) and (23) into (13), we have

$$
F_{R_{d}}\left(R_{0}\right) \doteq \rho^{-(N-1+\mu)} .
$$

Together with (21) and (24), we have from (16)

$$
O_{p} \doteq \rho^{-(N-1+\mu)} .
$$

Thus Theorem 1 is proved.

\section{REFERENCES}

[1] Z. Zhang, X. Chai, K. Long, A. V. Vasilakos, and L. Hanzo, "Full duplex techniques for $5 \mathrm{G}$ networks: Self-interference cancellation, protocol design, and relay selection," IEEE Commun. Mag., vol. 53, no. 5, pp. 128-137, May 2015.

[2] Z. Zhang, K. Long, A. V. Vasilakos, and L. Hanzo, "Full-duplex wireless communications: Challenges, solutions, and future research directions," Proceedings of the IEEE, vol. 104, no. 7, pp. 1369-1409, Jul. 2016.

[3] L. Li, H. V. Poor, and L. Hanzo, "Non-coherent successive relaying and cooperation: Principles, designs, and applications," IEEE Commun. Survey and Tutorials, vol. 17, no. 3, pp. 1708-1737, 3rd Quart. 2015.

[4] A. Sabharwal, P. Schniter, D. Guo, D. W. Bliss, S. Rangarajan, and R. Wichman, "In-band full-duplex wireless: Challenges and opportunities," IEEE J. Sel. Areas Commun., vol. 32, no. 9, pp. 1637-1652, Sept. 2014.

[5] T. Riihonen, S. Werner, and R. Wichman, "Mitigation of loopback selfinterference in full-duplex MIMO relays," IEEE Trans. Signal Process., vol. 59, no. 12, pp. 5983-5993, Dec. 2011.

[6] Y. Y. Kang, B.-J. Kwak, and J. H. Cho, "An optimal full-duplex AF relay for joint analog and digital domain self-interference cancellation," IEEE Trans. Commun., vol. 62, no. 8, pp. 2758-2772, Aug. 2014.

[7] T. Riihonen, S. Werner, and R. Wichman, "Hybrid full-duplex/half duplex relaying with transmit power adaptation," IEEE Trans. Wireless Commun., vol. 10, no. 9, pp. 3074-3085, Sept. 2011.

[8] N. H. Tran, L. J. Rodriguez, and T. Le-Ngoc, "Optimal power control and error performance for full-duplex dual-hop af relaying under residual self-interference," IEEE Wireless Commun. letters, vol. 19, no. 2, pp. 291-294, Feb. 2015.

[9] I. Krikidis, H. A. Suraweera, S. Yang, and K. Berberidis, "Full-duplex relaying over block fading channel: A diversity perspective," IEEE Trans. Wireless Commun., vol. 11, no. 12, pp. 4524-4535, Dec. 2012.

[10] T. Riihonen, S. Werner, and R. Wichman, "Mitigation of loopback selfinterference in full-duplex MIMO relays," IEEE Trans. Signal Process., vol. 59, no. 12, pp. 5983-5993, Dec. 2011.

[11] H. A. Suraweera, I. Krikidis, G. Zheng, C. Yuen, and P. J. Smith, "Lowcomplexity end-to-end performance optimization in MIMO full-duplex relay systems," IEEE Trans. Wireless Commun., vol. 13, no. 2, pp. $913-$ 927, Feb. 2014.

[12] A. Bletsas, A. Khisti, D. Reed, and A. Lippman, "A simple cooperative diversity method based on network path selection," IEEE J. Sel. Areas Commun., vol. 24, no. 3, pp. 659-672, Mar. 2006.

[13] A. Bletsas, H. Shin, and M. Z. Win, "Cooperative communications with outage-optimal opportunistic relaying," IEEE Trans. Wireless Commun., vol. 6, no. 9, pp. 3450-3460, Sept. 2007. 
[14] R. Tannious and A. Nosratinia, "Spectrally-efficient relay selection with limited feedback," IEEE J. Sel. Areas Commun., vol. 26, no. 8, pp. 14191428 , Oct. 2008.

[15] A. Adinoyi, Y. Fan, H. Yanikomeroglu, H. V. Poor, and F. AI-Shaalan, "Performance of selection relaying and cooperative diversity," IEEE Trans. Wireless Commun., vol. 8, no. 12, pp. 5790-5795, Dec. 2009.

[16] Q. Li, M. Yu, A. Pandharipande, and X. Ge, "Outage analysis of cooperative multi-path relay channels with virtual full-duplex relaying," IEEE ICC Workshop 2015, London, UK, Jun. 2015, pp. 925-930.

[17] Q. Li, M. Yu, A. Pandharipande, X. Ge, J. Zhang, and J. Zhang, "Performance of virtual full-duplex relaying on cooperative multi-path relay channels," IEEE Trans. Wireless Commun., vol. 15, no. 5, pp. 3628 3642, May 2016.

[18] I. Krikidis, H. A. Suraweera, P. J. Smith, and C. Yuen, "Full-duplex relay selection for amplify-and-forward cooperative networks," IEEE Trans. Wireless Commun., vol. 11, no. 12, pp. 4381-4398, Dec. 2012.
[19] J.-S. Han, J.-S. Baek, S. Jeon, and J.-S. Seo, "Cooperative networks with amplify-and-forward multiple-full-duplex relays," IEEE Trans. Wireless Commun., vol. 13, no. 4, pp. 2137-2149, Apr. 2014.

[20] Q. Li, M. Yu, A. Pandharipande, T. Han, J. Zhang, and X. Ge, "Cooperative two-path relay channels: Performance analysis using a markov framework," IEEE ICC 2015, London, UK, Jun. 2015, pp. 3573-3578.

[21] Q. Li, M. Yu, A. Pandharipande, and X. Ge, "Outage analysis of cooperative two-path relay channels," IEEE Trans. Wireless Commun., vol. 15, no. 5, pp. 3157-3169, May 2016.

[22] A. Bletsas, A. Lippnian, and D. P. Reed, "A simple distributed method for relay selection in cooperative diversity wireless networks, based on reciprocity and channel measurements," VTC 2005 Spring, pp. 1484-1488.

[23] T. M. Cover and J. A. Thomas, Elements of Information Theory. 2nd edition. Wiley, 2006. 